\title{
Anreicherungen, Mashups und Vernetzungen von Titeln in einem heterogenen Katalogverbund am Beispiel des Kölner UniversitätsGesamtkatalogs KUG
}

\author{
Oliver Flimm <flimm@ub.uni-koeln.de>
}

31.3 .2010

Der Kölner UniversitätsGesamtkatalog (KUG) besteht aus einer Vielzahl einzelner Katalogbestände, darunter allein 143 separate Institutskataloge. Aufgrund den dort vorherrschenden abweichenden Erfassungspraktiken, z.B. in der sachlichen Erschließung, müssen alternative Strategien angewendet werden, um für die Recherchierenden dennoch ein Höchstmaß an Homogenität in den ansonsten heterogenen Datenbeständen zu verwirklichen.

Diese Homogenisierung gilt sowohl für Verbesserungen der Recherchierbarkeit von Titeln, wie auch für deren Anreicherung mit weiteren interessanten Inhalten - kommen sie nun aus unserem eigenen System, von externen Diensten oder werden wiederum für externe Dienste nutzbar gemacht. Im KUG nähern sich so klassische Kataloganreicherung und Mashups einander an und führen im Interesse unserer Nutzer zu einer weitgehenen Vernetzung von Titeln, zusätzlichen Inhalten und verschiedenen Diensten.

Ermöglicht wird dies durch eine Kombination von a) Mashups mit gängigen externen Diensten, b) einer eigenen Anreicherungsdatenbank, in der die benötigten Vernetzungsinformationen und Inhalte für alle Katalogbestände gesammelt werden und c) einem Eingriff in die Synchronisation der Datenbestände des KUG mit den Erfassungssystemen, um auch jenseits der zentralen Anreicherungsdatenbank automatisch gezielt Inhalte in die einzelnen Kataloge einzuschleusen.

Die Anreicherungsdatenbank ist das zentrale Bindeglied zwischen den ansonsten einzelnen Katalogbeständen im KUG. Gerade sie bietet sich mit den dort enthaltenen Informationen als "Andockpunkt" für Mashups mit dem KUG durch von ihm bereitgestellte Konnektoren an. Ein Beispiel ist der Verfügbarkeits-Konnektor, der die Grundlage für eine Integration der KUG-Bestände in Amazon oder Google Books bildet.

Die Heterogenität der KUG-Bestände - ursprünglich ein Problem - ist so zu einer zentralen Voraussetzung geworden, um geeignete Strategien zu entwickeln und Stukturen für eine weitgehende Vernetzbarkeit zu schaffen. 


\section{Inhaltsverzeichnis}

1 Motivation 2

2 Die Bibliotheken der Universität zu Köln 2

3 Der „KUG“ als zentrales Nachweisinstrument 3

4 Automatisierte und zentrale Kataloganreicherung 4

Beispiel: Kataloganreicherung mit Schlagworten . . . . . . . . . . . . . . . . 6

$\begin{array}{lll}5 & \text { Vernetzung durch Mashups } & 8\end{array}$

Beispiel: Integration von BibSonomy . . . . . . . . . . . . . . . . . . . . . 10

6 Mashups durch externe Datenlieferungen 13

$\begin{array}{lll}7 & \text { Bereitstellung eigener Dienste und Daten } & 13\end{array}$

\begin{tabular}{lll}
\hline & Allgemeine Vernetzungen zwischen Titeln & 17
\end{tabular}

$\begin{array}{lll}9 & \text { Weitere Dienstleistungen aus den Daten der KUG-Plattform } & 17\end{array}$

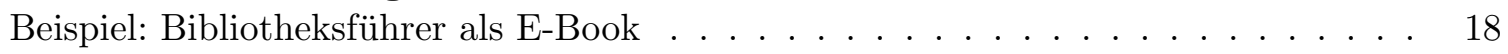

Beispiel: Liste der Zeitschriften in der ZDB $\ldots \ldots \ldots \ldots$. . . . . . . . . . 19

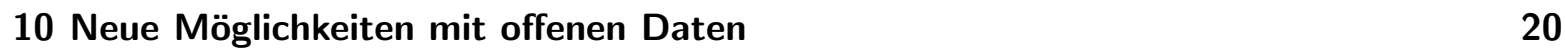

\begin{tabular}{ll}
\hline 11 Zusammenfassung & 21
\end{tabular}

\section{Motivation}

Der Katalog als Nachweisinstrument der in einer Bibliothek verfügbaren Medien gehört zu einer der zentralen Dienstleistungen für den Nutzer. Daher gilt es ihn zeitgemäß weiter zu entwickeln und dem Recherchierenden bessere und vielfältigere Möglichkeiten zu eröffnen, um an thematisch geeignete Literatur zu gelangen. Anhand des Kölner UniversitätsGesamtkatalogs KUG werden einige der dort umgesetzten Erweiterungen im Kontext allgemeiner Prinzipien und Techniken erläutert, mit denen die von der Bibliothek erbrachte Dienstleistung verbessert wurde.

\section{Die Bibliotheken der Universität zu Köln}

Die Universität zu Köln verfügt über ein sog. ,,zweischichtiges Bibliothekssystem“ Bauer 2004 in dem es neben einer zentralen Universitätsbibliothek, der Universitäts- und Stadtbibliothek Köln (USB Köln), eine Vielzahl an Instituts- und Seminarbibliotheken gibt. So kommt die Universität auf insgesamt mehr als 140 verschiedene Bibliotheken. 
Im Jahr 2001 wurde das Projekt „Kölner UniversitätsGesamtkatalog“ begonnen, um für den Endnutzer eine „funktionale Einschichtigkeit“ in Form eines zentralen Bestandsnachweises aller an der Universität elektronisch katalogisierten Medien zu erreichen. Dadurch wurden zusätzlich weitere Synergieeffekte zwischen den Bibliotheken sowie eine größere Homogenität in dieser hochgradig heterogenen Bibliothekslandschaft ermöglicht.

In diesem Projekt wurden die Institutsbibliotheken unter Federführung der USB Köln mit einem einheitlichen integrierten Bibliothekssystem ${ }^{1}$ ausgestattet, das von der USB betrieben und technisch über Citrix-Klienten in der Universität bereitgestellt wird. Auf einem einzigen Serversystem in der USB werden die einzelnen Bibliothekskataloge in separaten Datenbanken unter einer Installation der Bibliothekssystem-Software betrieben. Durch die Trennung können die dort verarbeiteten sensiblen Ausleih- und Erwerbungsdaten zwischen den einzelnen Bibliotheken bestmöglich geschützt werden.

\section{Der „KUG“ als zentrales Nachweisinstrument}

Als zentrale Recherche-Plattform der Katalogbestände für den Endnutzer - Wissenschaftler, Dozenten, Studenten und Bibliothekare - wird an der USB Köln die Open Source Portalsoftware OpenBib[Flimm 2007] als „Kölner UniversitätsGesamtkatalog“ oder kurz „KUG“ eingesetzt.

OpenBib ist in seiner Architektur ein klassisches „Schattensystem“, in dem die Recherche von den verschiedenen Erfassungssystemen strikt getrennt ist. Es verfügt über eine eigenständige Datenhaltung und speichert die bibliographischen Daten eines jeden Bibliothekskatalogs getrennt in einer eigenen relationalen Datenbank und einem eigenen Suchmaschinenindex ab. Bei der nächtlichen Aktualisierung der Datenbestände werden diese zunächst auf ein Zwischenformat vereinheitlicht und dann standardisiert weiterverarbeitet. In diesen Migrationsprozess kann eingegriffen werden, um die Daten automatisiert zu manipulieren.

Durch die Verwendung eines Zwischenformats lassen sich Daten sehr effizient aus beliebigen Erfassungssystemen integrieren, da lediglich ein Konvertierungsprogramm in das Zwischenformat erstellt werden muss. Trotz der Konsolidierung auf ein einheitliches Erfassungssystem ist diese Fähigkeit im Institutsbereich sehr wichtig. So katalogisieren einige unserer Institute originalsprachlich - z.B. das Ostasiatische Seminar mit seinen Abteilungen Japanologie und Moderne China Studien - und konnten daher mit ihren besonderen Schriftzeichen unsere vereinheitlichte Bibliothekssystemsoftware aufgrund dort fehlender UTF8-Fähigkeit bisher nicht verwenden. Der KUG unterstützt UTF8 und kann daher auch diese Kataloge problemlos wie all die anderen integrieren und so einen tatsächlichen Gesamtnachweis aller elektronisch erfassenden Institutsbibliotheken bereitstellen. Auch jenseits der Bibliotheksbestände werden in den KUG viele weitere relevante Datensammlungen integriert, wie z.B. der Hochschulschriftenserver der Universität, Research Papers in Economics, Digitalisate der Open Library uvm.

Der KUG stellt den Nutzern eine zeitgemäße moderne Rercherche-Technologie mit facettierter Suche und vielen weiteren Funktionen bereit. Mit Tagging und Literaturlisten werden die Anwender konsequent bei der Erstellung von Inhalten in die KUG-Plattform einbezogen und können so einen Mehrwert für alle anderen Anwender liefern. Bereits die einfache Nutzung des

${ }^{1}$ OCLC SISIS SunRise 
KUG genügt, um später durch geeignete Analysen des Anwenderverhaltens neue Inhalte zu erzeugen.

Eine der Grundstrategien im KUG ist die maximal mögliche thematische Vernetzung der Katalogdaten, sowohl innerhalb eines einzelnen, wie auch aller Kataloge - und zusätzlich mit weiteren externen Informationen. Dazu versuchen wir aktiv die Auffindbarkeit von Titeln durch den Nutzer zu verbessern. Durch den Einsatz eines Templating-Systems wird die Präsentation von Inhalten gesteuert und ist so sehr flexibel einsetzbar über verschiedene Abstraktionsebenen hinweg - Kataloge, Gruppen von Katalogen und die finale Präsentation in Sichten auf eben jene Kataloggruppen.

Aufgrund der Ausgangssituation mit vielen heterogenen Datenbeständen und der z.T. uneinheitlichen Erfassung in Bezug auf Verschlagwortung, Systematisierung oder die Vergabe von Medientypen, müssen für eine Vereinheitlichung und Anreicherung andere Wege beschritten werden, als bei einem einzelnen Katalog „im Vakuum“. Ebensowenig existiert das KUGGesamtsystem mit seinen vielen Datenbanken in einem Vakuum, sondern muss durch offene Schnittstellen für seine Dienste und Daten in das es umgebende Netz eingebettet werden.

\section{Automatisierte und zentrale Kataloganreicherung}

„Mit Kataloganreicherung (englisch catalog enrichment) werden Einträge eines Bibliothekskatalogs um weiterführende Informationen ergänzt, die über die reguläre Formal- und Sacherschließung hinausgehen. 22. Im Kontext der besonderen Situation eines Verbundes einzelner Erfassungssysteme werden insgesamt zwei Arten von Kataloganreicherungen im KUG verwirklicht.

Da ist zunächst eine automatisierte Anreicherung, die in den Prozess des Einspielens eines Katalogs in den KUG integriert ist. Durch diese Anreicherung werden die Daten für die Recherche durch den Nutzer verbessert.

Ein Problem ist hier beispielsweise die unvollständige Kennzeichnung der Titel mit rudimentären Medientypen wie Zeitschrift oder Aufsatz. Daher wird beim Neuaufbau eines Kataloges im KUG jeder Datensatz kategorieweise analysiert und anhand verschiedener Kriterien eben jene Medientypen bestimmt und hinzugefügt.

Ein anderes Problem liegt in besonderen Erfassungsspezifika und dem Umstand, dass ein gewöhnliches Recherchesystem einen Titelsatz lediglich anhand der in ihm vorkommenden Informationen finden kann. Im Schiller-Räuber-Problem ist dies aber nicht der Fall, da beide Informationen in verschiedene Titelsätze disjunkt verteilt sind - typischerweise „Schiller“ in der Hauptaufnahme „Werke“ und „Die Räuber“ in der untergeordneten Aufnahme ohne Nennung der Person „Schiller“. Daher muss in der untergeordneten Titelaufnahme auch die Person der übergeordneten Aufnahme für die Recherche herangezogen und indexiert werden.

Als letztes Beispiel sei die Problematik ISBN10/13 genannt, bei der Titel ehemals mit einer ISBN10 aufgenommen wurden, diese nun aber mit der zugehörigen ISBN13 recherchiert werden. Auch hier ist der Suchindex geeignet zu modifizieren, so dass unabhängig von der

\footnotetext{
${ }^{2}$ Wikipedia: http://de.wikipedia.org/wiki/Kataloganreicherung. Zuletzt besucht am: 26.3.2010
} 
ISBN-Variante der jeweilige Titel gefunden werden kann.

Bei der Anreicherung der Titel mit externen Inhalten bestehen in einem Katalogverbund wie dem KUG, mit vielen getrennten Katalogen, erschwerte Bedingungen. Einerseits ist der Aufwand, diese Informationen in die vielen - teils verschiedenen - primären Erfassungssysteme einzuladen und aktuell zu halten, sehr hoch. Andererseits lehnen einige Bibliothekare die „Verunreinigung" der von ihnen erfassten Katalogdaten tendenziell eher ab.

Daher haben wir diese Art der Anreicherung direkt in das KUG Rercherchesystem in Form einer separaten zentralen Anreicherungsdatenbank integriert. In dieser werden verschiedene Informationen zur Nutzung durch alle Kataloge abgelegt. Als Identifizierungsschlüssel dienen ISBN, ISSN bzw. der Bibkey ${ }^{3}$ - ein „bibliographischer Fingerabdruck“ des entsprechenden Titels.

$\mathrm{Zu}$ den zentral gesammelten Inhalten gehören zunächst einmal kategoriebasierte Inhalte. Die anzureichernden Informationen werden dazu unter einer numerischen Kategorie in der Datenbank abgelegt. Zusätzlich wird die Herkunft der Anreicherung kodiert hinzugefügt. Damit lassen sich gleichartige Inhalte aus verschiedenen Quellen nachträglich auseinander halten und besser aktualisieren. Ein Beispiel sind URL's auf universitätsweit lizensierte E-Books, die mit ihrer Print-ISBN13 dort in der Kategorie 4120 abgelegt werden.

Weiterhin wird zentral ein katalogübergreifender Gesamtnachweis aller Titel aus allen getrennten Katalogen aufgebaut. Um auf einen Blick die Existenz des Titels und die Zugehörigkeit $\mathrm{zu}$ einem bestimmten Katalog festzustellen und somit eine Anreicherung auch wieder in den Kontext des lokalen Katalogbestandes zu rücken, werden sowohl die Identifikationsnummer als auch der Katalogname eines jeden Titels unter der ISBN, ISSN bzw. dem Bibkey in der Datenbank abgelegt.

Zusätzlich sind dort die Gesamtnachweise aller ISBN's zu einem Werk angesiedelt: Alle ISBN's eines Werkes, also derselbe Titel in verschiedenen Ausgaben, Sprachen usw., werden in der Datenbank abgelegt. Als Datenlieferanten nutzt der KUG LibraryThing und die ThingISBN ${ }^{4}$.

Schließlich wird dort auch noch ein katalogübergreifender Gesamtnachweis der Ansetzungsformen von Normdaten geführt, um dem Nutzer z.B. effizient Suchvorschläge für seine Recherche aufgrund des tatsächlichen Bestands anbieten oder einen effizienten katalogübergreifenden Normdaten-Index verwirklichen zu können.

Der Vorteil dieser zentralen Anreicherungsdatenbank ist, dass die Anreicherungsinformationen nun nicht mehr mit einem speziellen Titel in einem speziellen Katalog verknüpft sind, sondern stattdessen von diesen getrennt - "freischwebend" - gehalten werden und dadurch automatisch nutzbar für alle Kataloge über die ISBN, ISSN bzw. den Bibkey sind. Der jeweilige Titel in irgendeinem Katalog „weiß“ selbst nichts von einer möglichen Anreicherung seiner bibliographischen Daten.

Erst bei der Einzeltrefferanzeige werden für einen konkreten Titel Katalog- und Anreicherungsdaten kombiniert und dann ausgegeben. In diesem Sinne stellt die im KUG verwendete zentrale

\footnotetext{
${ }^{3}$ http://www.gbv.de/wikis/cls/Bibliographic_Hash_Key. Zuletzt besucht am: 26.3.2010

${ }^{4}$ http://www.librarything.com/thingology/2006/06/introducing-thingisbn_14.php. Zuletzt besucht am: 26.3.2010
} 
Kataloganreicherung eine Form eines „internen Mashups“ dar.

Für eine effiziente Anreicherung bei der Recherche können gezielt beliebige Anreicherungsinhalte in den Suchindex eines Kataloges übernommen werden. Ein gutes Beispiel hierfür ist die Anreicherung eines Titels mit den Namen der Artikel, die ihn in der Wikipedia zitieren. Auf diese Weise werden dem Titel weitere Informationen für die Recherche hinzugefügt und er kann vom Endnutzer besser gefunden werden. Der Titel „Vergleichende Primatologie“ von Thomas Geissmann wird so z.B. für die Recherche um unzählige Namen von Affenarten erweitert.

\section{Beispiel: Kataloganreicherung mit Schlagworten}

In den verschiedenen Katalogen des KUG trifft der Endnutzer auf eine höchst unterschiedliche sachliche Erschließung. Für den gleichen Titel werden entsprechend dem verwendeten Regelwerk - die USB verschlagwortet nach RSWK - oder den Vorlieben der jeweiligen InstitutsbibliothekarInnen - dort wird frei, aber oft näher am Fachgebiet verschlagwortet - verschiedene Schlagworte vergeben. Dazu kommt, dass im besten Fall immerhin noch verschiedene, im schlimmsten Fall jedoch gar keine Schlagworte vergeben werden. Einmal abgesehen von der verwirrenden und uneinheitlichen Erscheinungsform des Titels in der Vollanzeige für den Recherchierenden, kann dieser den Titel in einem Katalog eventuell über die Suche nach dem entsprechenden Schlagwort finden, im anderen aber gerade nicht.

Dieses Problem lässt sich sehr einfach mit der zentralen Kataloganreicherung im KUG lösen.

Dazu werden in einem ersten Schritt bei der nächtlichen Aktualisierung eines jeden Katalogs im KUG die dort vergebenen Schlagworte mit der zugehörigen ISBN als Zugriffsschlüssel in unserer zentralen Anreicherungsdatenbank abgelegt. Damit wurden bereits folgende zwei Ziele erreicht. Zunächst stehen nun jeder Titelaufnahme über ihre ISBN alle korrespondierenden "angereicherten“ Schlagworte jenseits der (etwaig vorhandenen) lokalen Verschlagwortung zur Verfügung. Zusätzlich wird durch die Anreicherungdatenbank ein Verknüpfungsnetz von Titeln, identifiziert durch alle ISBNs zum jeweiligen Schlagwort - quasi unsichtbar - über den aktuellen (Teil-)Katalog gelegt. Auf diese Weise lassen sich zu jedem so angereicherten Schlagwort auch weitere thematisch gleich eingeordnete Titel im aktuellen Katalog finden und verknüpfen - auf Grundlage der Verschlagwortung des Titels in einem anderen Katalog.

Im KUG werden diese „angereicherten Schlagworte“ mit entsprechender Verknüpfung in der Einzeltrefferanzeige im Block "Entdecken Sie weitere Treffer über:" als „Verschlagwortung aus anderen Katalogen" angezeigt - für den Recherchierenden bewusst getrennt von den sonstigen bibliographischen Daten.

In einem zweiten Schritt werden nun nur noch durch entsprechende Parametrisierung die „angereicherten Schlagworte" mit in den Suchindex des jeweiligen Katalogs übernommen und sind so neben den „normalen“ Schlagworten recherchierbar. Ein derart mit weiteren Schlagworten angereicherter Titel profitiert unmittelbar von dieser Anreicherung, weil die Wahrscheinlichkeit steigt, dass er „mit den Suchworten des Recherchierenden“ auch gefunden wird. Die Grundannahme ist also: Je größer die Wortbasis der intellektuell verschlagwortenden BibliothekarInnen, desto größer auch die Wahrscheinlichkeit, dass der Nutzer mit einem davon recherchiert und dadurch den Titel findet. 


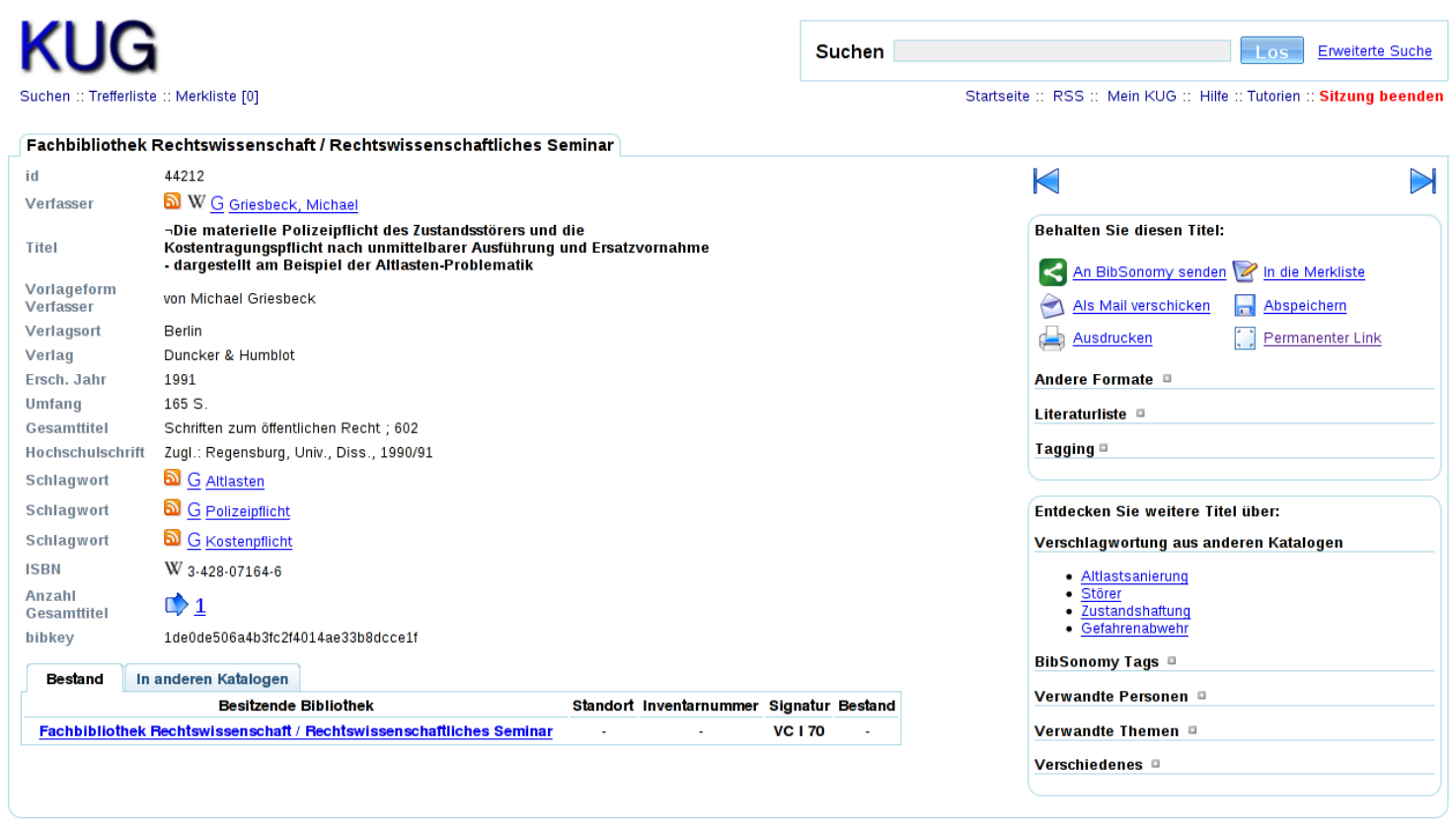

Abbildung 1: Beispiel für die Integration der Verschlagwortung in anderen Katalogen

Ein gutes Beispiel für diese Kataloganreicherung mit Schlagworten ist der Titel „Die materielle Polizeipflicht des Zustandsstörers und die Kostentragungspflicht nach unmittelbarer Ausführung und Ersatzvornahme - dargestellt am Beispiel der Altlasten-Problematik 45 von Michael Griesbeck aus dem Katalog der Fachbibliothek Rechtswissenschaft (Abbildung 1).

Dieser Titel wurde lokal mit den Worten Altlasten, Kostenpflicht und Polizeipflicht verschlagwortet. Durch die Anreicherung kommen nun noch die Begriffe Altlastsanierung, Störer, Zustandshaftung und Gefahrenabwehr hinzu.

Gleichzeitig ist dieser Titel auch im Katalog des Instituts für Öffentliches Recht und Verwaltungslehre vorhanden.

Dort ist der Titel überhaupt nicht verschlagwortet und er profitiert maximal von der Anreicherung. Mehr noch - wie bereits angesprochen können alle Titel durch den Nutzer in diesem Katalog erreicht werden, die auch in anderen Katalogen vorhanden sind und dort entsprechend verschlagwortet wurden - z.B. mit dem Schlagwort Gefahrenabwehr ergeben sich so 5 zusätzlich vernetzte Titel.

Die Kataloganreicherung mit Schlagworten ist ein gutes Beispiel dafür, wie mit relativ wenig Aufwand für den Recherchierenden ein deutlicher Mehrwert im Bereich Recherchierbarkeit sowie thematische Titelvernetzung geschaffen werden konnte.

Zusätzlich zu den intellektuell erfassten bibliographischen Daten und ihrer Erschließung kann sich die Auffindbarkeit von Titeln durch die automatische Verarbeitung verschiedener titelbezogener Informationen wie Inhaltsverzeichnisse, Register und Glossare anhand linguistischer

\footnotetext{
${ }^{5}$ PermaLink: http://kug.ub.uni-koeln.de/portal/connector/permalink/inst201/44212/1/kug/index.html. Zuletzt besucht am: 26.3 .2010
} 
sowie semantischer Methoden weiter verbessern lassen. Eine Erweiterung des KUG um solche Techniken steht zur Zeit noch aus.

Der Einsatz der Kataloganreicherung - automatisch und zentral - stellt ein wesentliches Werkzeug in einem Verbund unabhängiger Kataloge dar, um für den Nutzer - neben dem Zugewinn an Auffindbarkeit und Information - eine Homogenität zwischen den Katalogen zu schaffen, die ursprünglich gar nicht existierte.

\section{Vernetzung durch Mashups}

Unter einem Mashup versteht man die „Erstellung neuer Medieninhalte durch die nahtlose (Re-)Kombination bereits bestehender Inhalte $\sqrt[6]{6}$. Im Web 2.0 gehören sie zu einer häufig genutzten Technik, um externe Dienste mit ihren Inhalten in die eigene Anwendung unter Verwendung von Zugriffsschnittstellen (API's) einzubinden. Der externe Dienst wird über das Internet direkt angesprochen und liefert seine Informationen zurück.

Häufig geschieht dies durch den Einsatz von JavaScript und AJAX direkt auf einer Webseite und damit effektiv im Browser des Endnutzers. Die Verknüpfung und Verarbeitung externer Informationen kann aber auch in die jeweilige Anwendung integriert sein. Dann kommuniziert die eigene Anwendung mit dem Dienst und verarbeitet die Informationen danach selbst intern weiter.

Die Nutzung eines externen Dienstes über einen Mashup stellt einen sehr effizienten Mechanismus dar, da der Aufwand entfällt, diesen Dienst selbst lokal zu implementieren und somit das Rad neu zu erfinden.

Einer der bekanntesten und am häufigsten genutzten Dienste ist Google Maps für die Integration geographischen Kartenmaterials. Andere Beispiele sind Flickr, YouTube oder SlideShare.

Auch im Kontext von Bibliothekskatalogen kann die Technik von Mashups sinnvoll angewendet und so ein Mehrwert für den Endnutzer erreicht werden Hahn Schulze 2009] Stelzenmüller 2008.

Ebenso wie bei der automatischen und zentralen Kataloganreicherung im KUG können Mashups direkt für alle Kataloge eingesetzt werden und sind daher gerade in unserem Verbund separater Kataloge ein sehr geeignetes Werkzeug einen Mehrwert bei gleichzeitig geringem Arbeitsaufwand zu liefern. Dazu reicht es i.A. aus, ein entsprechendes JavaScript-Fragment in einem geeigneten katalogübergreifend genutzten Ausgabe-Template abzulegen.

Als Alternative für den Einsatz von JavaScript werden einige Inhalte auch über einen eigenen KUG-Dienst AvailabilityImage ausgegeben, der Verfügbarkeiten in externen Systemen über ein Bild signalisiert.

Dieses „Bild“ wird über einen URL verlinkt, der die Recherche bzw. den Einsprung in den jeweiligen externen Dienst aufruft - üblicherweise mit der in der Titelaufnahme enthaltenen ISBN. Das „Verfügbarkeits-Bild“ wird dann dynamisch über den lokalen AvailabilityImageDienst erzeugt.

\footnotetext{
${ }^{6}$ Wikipedia: http://de.wikipedia.org/wiki/Mashup_(Internet). Zuletzt besucht am: 26.3.2010
} 

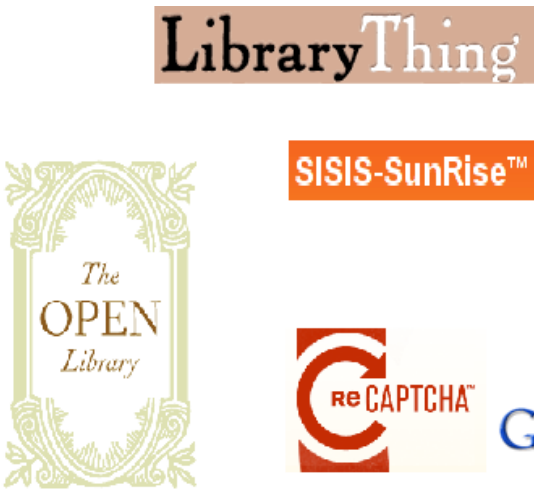

\section{CONTENTdm ${ }^{\otimes}$} Digilal Cullection Marragemenıl Sullware
BibSonomy

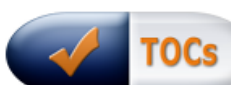

Google bücher

OpenDIA

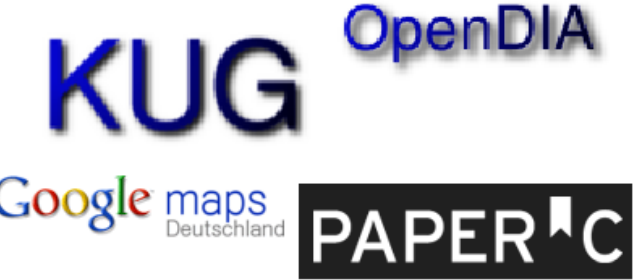

Simplified

Content Modelling

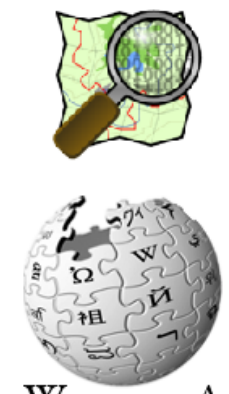

WIKIPEDIA

Die freie Enzyklopädie

E Z B Elektronische 000 Zeitschriftenbibliothek

Datenbank-Infosystem (DBIS)

Abbildung 2: Konsequente Nutzung externer Dienste im KUG

Dieser überprüft - ebenfalls über eine einfache Recherche - die Verfügbarkeit von Informationen über den aktuellen Titel im entfernten System. Existieren Informationen zum aktuellen Titel, so wird ein geeignetes Status-Bild per internem Redirect ausgegeben und der Recherche-Link ist nutzbar. Im anderen Fall wird stattdessen ein transparenter Pixel ausgegeben und der Link ist unsichtbar. Dieses sehr einfache Verfahren hat sich insbesondere in den Fällen bewährt, wenn gerade (noch) kein API vorhanden ist, wie es z.B. bis März 2010 beim „Internet-CopyShop" PaperC der Fall war. Insgesamt hat sich jedoch der Einsatz von API's durchgesetzt.

Weit verbreitet bei API's sind hier Umsetzungen mit REST (Representational State Transfer), XML-RPC und SOAP sowie JSON bzw. XML als Format für die Antwort auf eine Anfrage.

Im KUG versuchen wir die Möglichkeiten, die das Netz mit seinen Diensten bietet, konsequent zu nutzen (Abbildung 2).

Google Bücher liefert weitergehende Informationen wie ausführliche Inhaltsbeschreibungen und Inhaltsverzeichnisse, Verweise auf das aktuelle Buch in anderen Büchern oder Artikeln usw.

Google Maps liefert Kartenmaterial, so dass im Kontext allgemeiner Informationen zu einer Bibliothek - wie sie an der USB im sog. Bibliotheksführer für alle Bibliotheken an der Universität zu Köln erfasst werden - innerhalb des KUG auch die Position der jeweiligen Bibliothek auf einem Kartenausschnitt verzeichnet werden kann.

Open Library liefert in Kombination mit Google Bücher für den KUG Bilder von Buch-Covern. TicTocs/JOURNAL TOCs liefert eine Übersicht der zuletzt in einer Zeitschrift veröffentlichten Artikel, teilweise mit Inhaltszusammenfassung und direkter Verlinkung des Artikels.

ReCaptcha schützt die freie Registrierung von Endnutzern für den KUG vor automatisiertem Zugriff und dem damit verbundenen Missbrauchspotential. Über PaperC können Bücher kostenfrei online gelesen werden.

Und schließlich werden die Elektronische Zeitschriftenbibliothek EZB und das Datenbankin- 
formationssystem DBIS über thematisches Browsing und die gezielte Suche nach Zeitschriften sowie Datenbanken eingebunden.

\section{Beispiel: Integration von BibSonomy}

Aufgrund seiner konzeptionellen Bedeutung, die deutlich über die bisher genannten Mashups hinaus geht und als Beispiel für einen direkt in die KUG-Plattform integrierten Dienst wird nun auf das „Social bookmark and publication sharing system,, BibSonomy $]^{7}$ näher eingegangen.

Bereits Anfang 2007 wurde der KUG um eine eigene Tagging-Funktion erweitert. War diese zunächst noch eine zeitgemäße Alternative zu klassischen strukturierten Merklisten, wurde wenig später durch die Öffnung öffentlich markierter Tags für andere auch eine gemeinschaftliche Nutzung ermöglicht. Dieses Social-Tagging [Voß 2007] [Tonkin et al 2008] auf lokaler Ebene bringt jedoch auch Probleme mit sich.

Das größte Problem besteht in der lokal nicht erreichten kritischen Masse und Zersplitterung der Nutzer. Die potentielle Nutzerschaft für einen lokalen Katalog ist typischerweise begrenzt. Für ein erfolgreiches Social-Tagging ist aber eine kritische Masse an Endanwendern notwendig, die entsprechend aktiv ist. Durch die Existenz diverser Bibliothekskataloge - eine Universität, eine Bibliothek, ein Katalog - findet aber zwangsläufig eine Zersplitterung statt. Der mögliche erreichbare Nutzen ist nicht optimal.

Darüber hinaus werden die lokal vergebenen Tags ein Datensilo im Rechercheportal. Zwar sind die Katalogdaten durch vielfältige Exportmöglichkeiten 'befreit', die lokal vergebenen Tags sind aber in der Rechercheanwendung eingeschlossen und bilden so ein eigenes Datensilo. Auch diesen Umstand gilt es zu verbessern.

Vor diesem Hintergrund ist es sehr ratsam, die lokale Rechercheplattform mit anderen Systemen zu kombinieren, die dann als Datenaggregator auftreten und über eine entsprechend hohe Nutzerzahl verfügen, mit der sie die so wichtige kritische Masse erreichen.

Typische Beispiele für solche Dienste sind BibSonomy und Connotea und auch das ursprünglich nur lokal nutzbare Zotero beschreitet den Weg zu einer vielversprechenden kollaborativen Plattform.

Für den KUG fiel die Wahl auf BibSonomy. Dieses System verfügt über ein eigenes API und kann daher ideal als Mashup in den KUG eingebunden werden.

Neben der - schon vor dem Einsatz des BibSonomy-API existierenden - Möglichkeit im KUG einzelne Titel gezielt nach BibSonomy zu übertragen, können nun - mit dem API- alle bibliographischen Nachweise und Web-Quellen in BibSonomy vollständig mit einem thematischen Browsing über Tags und Schlagworte erschlossen werden. Darüber hinaus findet eine „Spiegelung der lokalen Tagging-Aktionen" in BibSonomy statt.

Hintergrund des „thematischen Browsens“ ist der Wunsch, über die zentrale Datenbasis von BibSonomy anhand von Tags oder Schlagworten weitere thematisch infrage kommende Quellen - Publikationen und Bookmarks - zu entdecken. Damit wird dem Aspekt Serendipity „Ich

${ }^{7}$ http://www. bibsonomy.org/. Zuletzt besucht am: 26.3.2010 


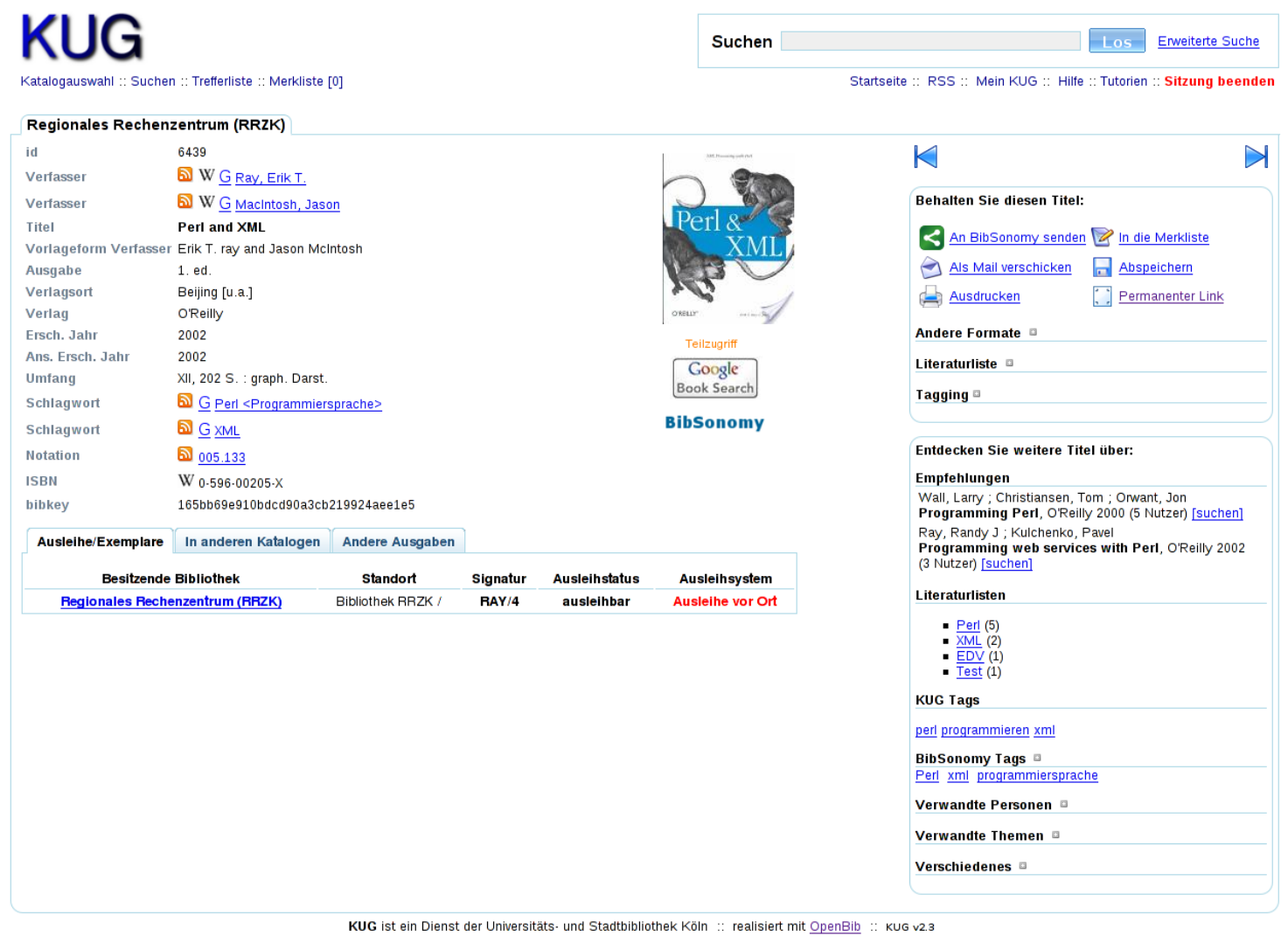

Abbildung 3: Beispiel für die Verbindung eines Titels zu BibSonomy anhand von Tags und Schlagworten

möchte auch etwas finden, was ich gar nicht gesucht habe ${ }^{8}$ Rechnung getragen.

Dazu wird zunächst bei der Einzeltrefferanzeige eines Titels im KUG mit dem Bibkey (konkreter: dem derzeitigen inter-hash key ${ }^{9}$ von BibSonomy) eine Abfrage in BibSonomy gemacht und der aktuelle Titel gesucht. Alle Tags, die gegebenenfalls beim dort gefundenen Titel vergebenen wurden, können dann anschließend im KUG unter dem Abschnitt „BibSonomy Tags“ ausgegeben werden.

Da nicht jeder Titel aus dem KUG schon in BibSonomy vorhanden ist, werden zusätzlich die im KUG-Titel verwendeten Schlagworte „als Tags“ mit BibSonomy abgeglichen (Abbildung 3).

Auf diese Weise erhält der Nutzer schließlich eine Aufstellung valider Tags, über die er in den Bestand von BibSonomy eintauchen kann und eine Aufstellung dort vorhandener Publikationen bekommt (Abbildung 4). Dieser automatische Abgleich mit BibSonomy ist vollständig in die KUG-Oberfläche eingebettet.

\footnotetext{
${ }^{8}$ Beluga Blog: http://beluga-blog.sub.uni-hamburg.de/blog/2008/02/22/wenn-das-klima-anders-waere -koennte-man-auch-eine-unfertige-liste-oeffnen-beluga-in-der-diskussion/. Zuletzt besucht am: 26.3.2010

${ }^{9}$ http://www.bibsonomy.org/help/doc/inside.html. Zuletzt besucht am: 26.3.2010
} 


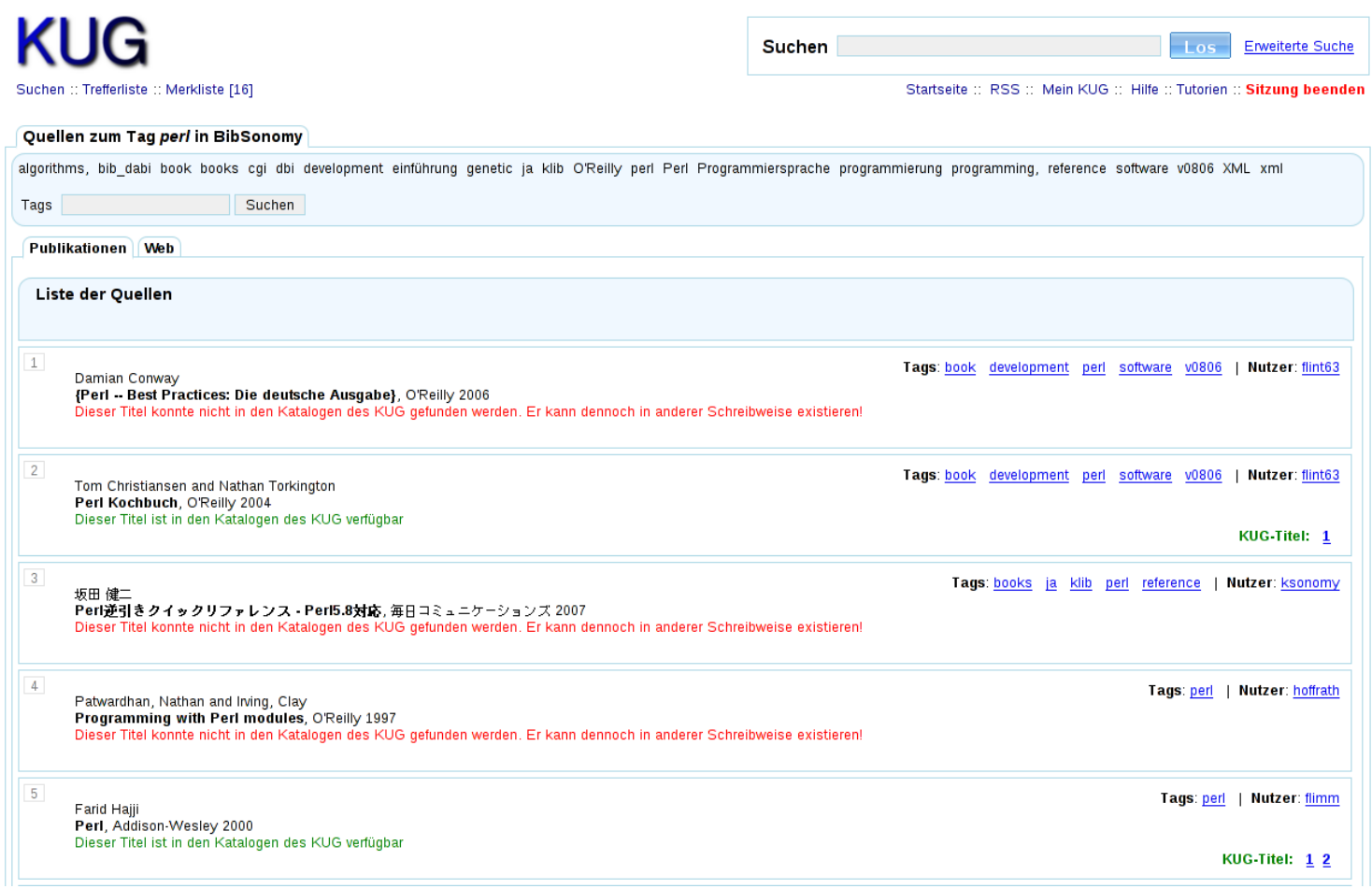

Abbildung 4: Titel zum Tag „perl“ in BibSonomy mit weiterer Vernetzung von Tags und Nutzern

Anhand der Bibkeys für die in BibSonomy gefundenen Publikationen wird dann augenblicklich wieder eine Verfügbarkeitsabfrage im KUG-Bestand gemacht, so dass für die durch BibSonomy gelieferten Titel sofort angezeigt werden kann, ob sie im KUG-Bestand vorhanden sind. Grundlage hierfür ist die vorherige Anreicherung der KUG-Titel mit Bibkeys sowie der Gesamtnachweis in der zentralen Anreicherungsdatenbank.

Stellt das „thematische Browsing“ einen Weg dar, BibSonomy in den eigenen Katalog zu integrieren und als die angesprochene „gemeinsame“ Datenbasis lokal zu nutzen, wird mit der vollautomatischen Spiegelung der lokalen Tagging-Aktionen nach BibSonomy genau die dazu notwendige Öffnung des Datensilos OPAC und ein verbesserter (Katalog- und Tagging-) Datenfluss nach BibSonomy forciert.

Dazu taggt der Endnutzer direkt im KUG. Seine Tags und Titel werden durch den BibSonomyMashup nun aber nicht mehr nur im KUG gespeichert. Darüber hinaus werden die Titel im KUG - so sie im BibSonomy-Account des Nutzers noch nicht vorhanden sind - automatisch dort eingetragen und mit den lokal im KUG vergebenen Tags und der Sichtbarkeitsinformation (öffentlich oder privat) versehen. Ändert er seine Tags im KUG, so werden diese Änderungen auch in BibSonomy automatisch nachgezogen.

Auf diese Weise können die Nutzer weiterhin lokal das Rechercheportal mit all seinen Funktionen nutzen, die Daten wandern aber zusätzlich zur gemeinsamen 'Datenzentrale' BibSonomy. Jenseits der weitergehenden Funktionen von BibSonomy für den Nutzer, erhält er zusätzlich - rückgekoppelt im lokalen Kontext - weitere Verweise auf Literatur, die an der Universität 
vorhanden ist.

\section{Mashups durch externe Datenlieferungen}

Bisher wurden Mashups vorgestellt, die sich sofort über das Netz mit einem externen Dienst verbinden, dort Informationen sammeln und mit der lokalen Anwendung zu etwas Neuem kombinieren. Einige Dienste bieten jedoch auch die Möglichkeit an, die Gesamtheit der Informationen direkt herunterzuladen. Die lokale Abspeicherung und Verarbeitung dieser Informationen bietet dann u.a. zusätzlich die Möglichkeit, diese Informationen auch für alle lokal vorhandenen Titel in Nutzerrecherchen zu verwenden.

Die Verarbeitung solcher externer Daten findet im KUG zentral in der Anreicherungsdatenbank statt. Typische Beispiele wurden bereits im entsprechenden Abschnitt über die zentrale Kataloganreicherung genannt. Es sind LibraryThing mit der ThingISBN für die Gesamtnachweise aller ISBN's zu einem Werk sowie die Wikipedia für die Namen der Artikel, in denen auf Titel mit deren ISBN verwiesen wird.

\section{Bereitstellung eigener Dienste und Daten}

Ebensowenig wie ein einzelner Katalog im Vakuum ohne die anderen Kataloge im KUG gesehen werden kann und man daher katalogübergreifend denken muss, steht auch der KUG nicht für sich allein, sondern ist in eine Welt aus Diensten und Daten eingebettet. Dementsprechend kommt zum „katalogübergreifenden Denken“ ein „systemübergreifendes Denken“ hinzu.

So hat sich der KUG über die Jahre von einer reinen 1-dimensionalen Recherchelösung im klassischen OPAC-Sinn hin zu einem Portal-Baukasten und einer allgemeinen Infrastrukturlösung an der USB Köln entwickelt, die sowohl Dienste wie auch Daten zur externen Verwendung bereit stellt. Der KUG ist nicht mehr nur ein Katalog, sondern eine vielfältig nutzbare Plattform.

Dazu tragen speziell die verschiedenen Organisationsmöglichkeiten für einzelne Kataloge in der KUG-Plattform bei. In nur einer Installation des KUG lassen sich aus der Gesamtmenge aller Kataloge beliebige Gruppen in eigenen Katalog-Profilen zusammenfassen. Diese Profile lassen sich dann wiederum über sog. Sichten in verschiedenen Präsentationen für den Endnutzer aufbereiten und bilden dann ein eigenständig ansprechbares Portal.

Diese drei verschiedenen Abstraktionsebenen im KUG - Datenbanken, Sichten und Profile (zur Gruppierung von Datenbanken/Sichten) - können bequem über ein Web-Frontend verwaltet werden und übertragen sich exakt auf das für die Web-Präsentation eingesetzte objektorientierte Templatingsystem.

An der USB Köln können durch die so erreichte Flexibilität ohne viel Aufwand - jenseits der Standard-KUG-Sicht mit fast allen verfügbaren Katalogen - getrennte Varianten für jedes Institut sowie eigenständige Projekt- und Themenportale eingerichtet werden. Im März 2010 summierte sich die Gesamtzahl der von der KUG-Plattform bereitgestellten individuellen Portal-Sichten auf 183. 


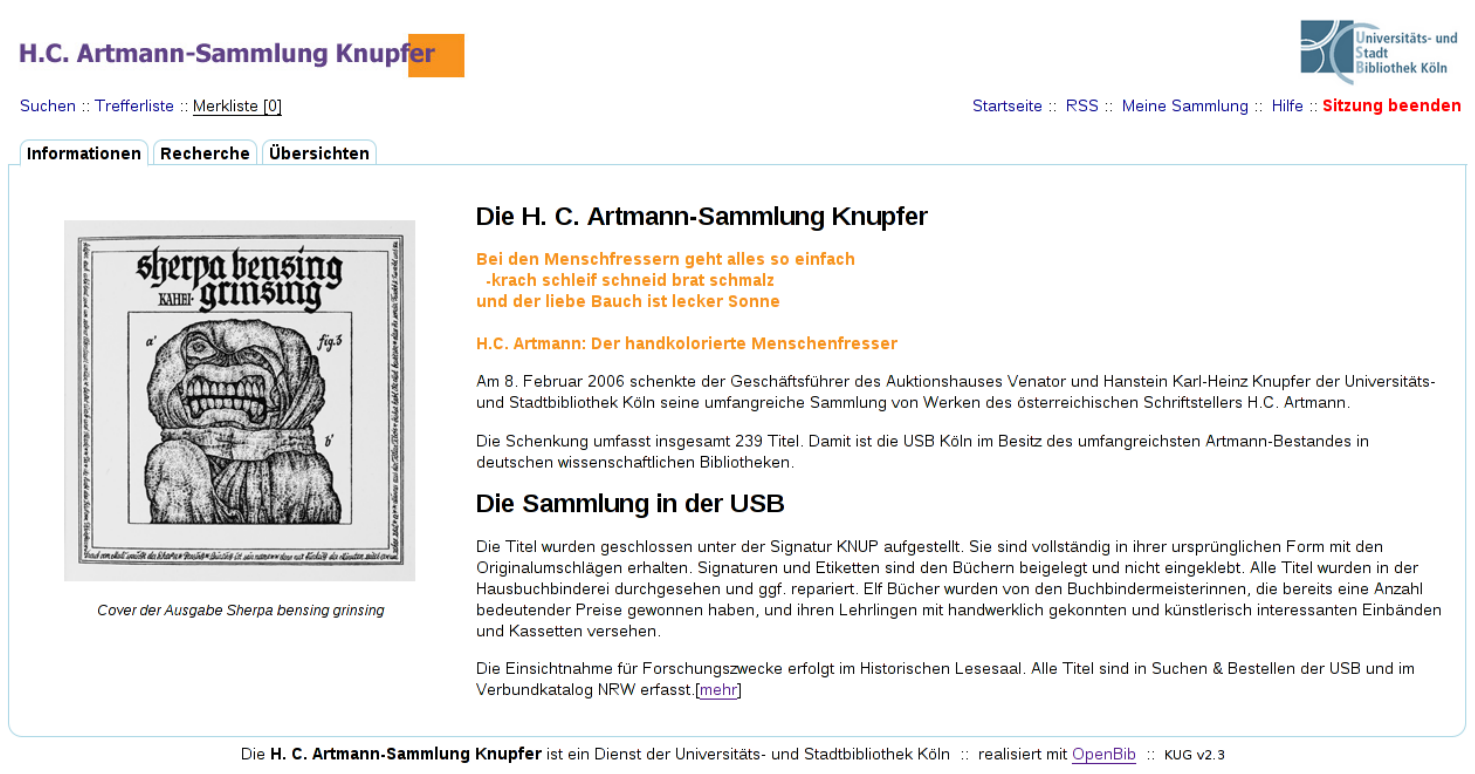

Abbildung 5: Die H. C. Artmann-Sammlung Knupfer als Beispiel für ein eigenständiges Portal in der KUG-Plattform

Ein gutes Beispiel hierfür sind die vielen Sammlungen der Universitäts- und Stadtbibliothek Köln, die jedoch im Gesamtbestand aller Titel im Katalog der USB leider weitgehend untergehen - vielen Nutzern erschließt sich nicht, welche Sammlungen die USB überhaupt besitzt.

Aus diesem Grund hat die USB begonnen, Kriterien für deren Identifikation zu bestimmen, um dann in einem ersten Schritt die jeweilige Sammlung aus dem Gesamtbestand zu extrahieren und in eine eigene Datenbank in der KUG-Plattform einzuspielen. Die Sammlungsbestände werden so - jeder für sich - aus der Anonymität des gesamten Kataloges herausgelöst und getrennt ansprechbar.

Dazu nutzen wir einfache Konvertierungs-Plugins, die für die Bildung der jeweiligen Teilbestände aus dem USB Katalog sorgen. Sehr vorteilhaft ist hier wieder die eigenständige Datenhaltung in einer relationalen Datenbank und einem Suchmaschinenindex in der KUGPlattform, mit der Sammlungen auch aus verschiedenen Erfassungssystemen dort zusammengeführt werden können. Das ist umso wichtiger, da etliche Sammlungen an der Universität zu Köln gerade nicht im USB-Katalog nachgewiesen sind, wir aber eine Gesamtlösung für die Universität anstreben. Das Spektrum der vorhandenen Daten reicht hier von der einfachen Excel-Datei bis zum spezialisierten Erfassungsystem.

Für jede dieser Sammlungen wird eine eigene Portal-Sicht erstellt, in der die Bestände beschrieben und über Recherchemöglichkeiten, Übersichten und/oder Register für die Nutzer zugänglich gemacht werden. Mit dieser individuellen Präsentation möchten wir unserer Wertschätzung einer jeden Sammlung und eines jeden Sammlers Ausdruck verleihen und unsere Nutzer auf diese verborgenen Schätze aufmerksam machen (Abbildung 5).

Neben der Fähigkeit getrennte Portale einzurichten stellt die KUG-Plattform viele ihrer Funktionen auch durch eigene offene Schnittstellen - den sog. Konnektoren - für eine externe Nutzung 
bereit. Dies geschieht im Allgemeinen in Form von Web Services. Damit lassen sich Konnektoren ideal zum Aufbau serviceorientierter Infrastrukturen auf Basis des KUG nutzen.

Über den DigiBib-Konnektor, dessen Abfrageprotokoll auf Vorgaben des Hochschulbibliothekszentrums NRW (hbz) beruht, können verschiedene Kataloge sowohl in unser lokales USB-Portal wie auch mit dem Literaturverwaltungsprogramm Citavi durchsucht werden. Hier wurde vom hbz bewusst einem einfachen HTML-basierten Abfrageprotokoll gegenüber dem komplexeren Standard Z39.50 der Vorzug gegeben, das einfach implementier- und erweiterbar ist - z.B. um Facetten bei der Verwendung von Suchmaschinentechnologie.

SeeAlso Voß 2008 ist ein Abfrageprotokoll, das von Jakob Voß und der GBV Verbundzentrale zur Anreicherung von Rechercheergebnissen erstellt wurde und Links zu weiterführenden Informationen bereitstellt. Auch dieses Protokoll wird durch einen eigenen Konnektor im KUG bereitgestellt und derzeit primär für den Transport von Informationen aus unserer zentralen Anreicherungsdatenbank verwendet.

Ein sehr großer Vorteil von SeeAlso ist, dass sich darüber beliebige Informationen für eine Anreicherung der Titel in externen Katalogen transportieren lassen und eine Erweiterung sehr einfach möglich ist.

Beispiele für SeeAlso-Dienste, die der KUG bereit stellt sind

- isbn2wikipedia zur Lieferung der Namen der Artikel in der (deutschen) Wikipedia, die die jeweilige ISBN referenzieren,

- isbn2subject zur Lieferung der Schlagworte, die für den über die ISBN referenzierten Titel vergeben wurden,

- issn2tictocs zur Lieferung von RSS-Feeds mit Informationen über die aktuellsten Artikel einer Zeitschrift (basierend auf dem TicTocs-Dienst),

- thingisbr ${ }^{10}$ zur Lieferung aller anderen ISBN's zu den verschiedenen Erscheinungsformen des Werks sowie

- isbn2kug zur Lieferung von Katalogen und PermaLinks zu den Titeln im KUG.

Ein weiterer Availability-Konnektor liefert zu einer ISBN ausführliche Verfügbarkeitsinformationen der entsprechenden Titel in den Katalogen des KUG. Neben der Information über besitzende Bibliotheken samt PermaLink werden zusätzlich getrennt auch Nachweise der Titel in anderen Auflagen, Sprachen usw. geliefert.

Dieser Konnektor wird verwendet, um dem Endnutzer über die Firefox-Erweiterung GreaseMonkey in seinen Recherchen bei Amazon oder Google Bücher direkt die Verfügbarkeit des jeweiligen Titel im KUG anzuzeigen. Falls der Titel selbst nicht vorhanden ist, werden stattdessen die Nachweise der anderen Auflagen usw. ausgegeben. Auf diese Weise können die Nachweise lokaler Bestände dort angezeigt werden, wo sich der Nutzer befindet (Abbildung6).

Auch bei unseren Lieferanten wird dieser Konnektor im Rahmen der Vorakzession in Approval-

${ }^{10}$ Dieser Dienst darf entsprechend der Bedingungen von LibraryThing ausschließlich für nichtkommerzielle Zwecke verwendet werden. 


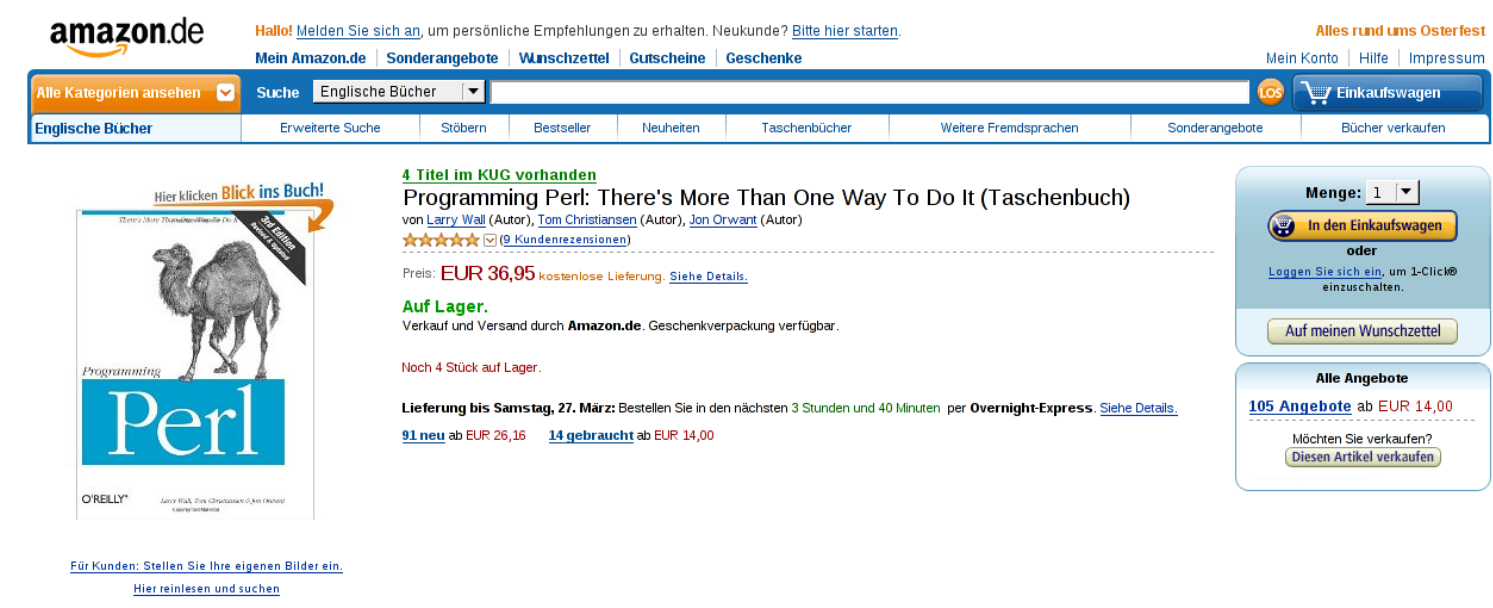

Abbildung 6: KUG-Bestand in Amazon: Katalognachweise dort anzeigen, wo der Nutzer ist

Plänen genutzt, um die Verfügbarkeit eines Titels nicht mehr händisch am USB-OPAC durchführen zu müssen, sondern automatisch über diesen Web-Service.

Für die Auslieferung maschinenlesbarer bibliographischer Informationen zu einzelnen Titeln verwendet der KUG die unAPI-Schnittstelle Chudnow et al 2006]. Über diese können die Titelinformationen - anders als bei COins 11 - in verschiedenen Formaten ausgegeben werden, wie z.B. BibTeX, MODS, DC - oder METS, wie im Fall von WikiSource.

Der KUG hat die bibliographischen Nachweise von Digitalisaten in WikiSource zusammen mit der Struktur der Digitalisate in einem eigenen Katalog abgelegt. Diese Strukturinformationen sind über unAPI im METS-Format abfragbar und können damit direkt an den DFG-Viewer ${ }^{12}$ zur Anzeige des Digitalisats übergeben werden. Durch die Kombination offener Daten von WikiSource, einem Mashup-fähigen Dienst der USB und einem der DFG konnte so für unsere Nutzer ein Mehrwert geschaffen werden, der das Potential von Mashups sehr klar verdeutlicht.

Aber auch andere Programme nutzen direkt die unAPI-Schnittstelle. Ein sehr prominentes Beispiel ist Zotero, ebenfalls eine Erweiterung des Web-Browsers Firefox. Mit Zotero kann der Nutzer via unAPI direkt aus der Einzeltrefferanzeige deren bibliographische Daten übernehmen.

Dies waren nur einige Beispiele, die aber zeigen, dass es nicht nur sinnvoll ist Mashups selbst zu nutzen, sondern dass es mindestens ebenso wichtig ist, sein eigenes System so anzupassen, damit dieses durch offene Schnittstellen auch selbst über Mashups eingebunden werden kann.

\footnotetext{
${ }^{11}$ OpenURL COinS: A Convention to Embed Bibliographic Metadata in HTML http://ocoins . info/ . Zuletzt besucht am: 26.3.2010

${ }^{12}$ http://dfg-viewer.de/ Zuletzt besucht am: 26.3.2010
} 


\section{Allgemeine Vernetzungen zwischen Titeln}

Bisher wurden bereits einige Beispiele für das Prinzip Serendipity „Sinnvolles finden, das gar nicht gesucht wurde“ angesprochen. Die Erweiterung des Kataloges um zusätzliche thematische Querverbindungen zwischen verschiedenen Titeln ist daher eine für den Endnutzer sehr wesentliche Eigenschaft einer Rechercheanwendung.

Daher versuchen wir im KUG fortlaufend aus den vorhandenen Daten möglichst automatisch weiterführende Informationen und Vernetzungen zwischen Titeln zu identifizieren und dann einer sinnvollen Nutzung zuzuführen.

Die allgemeinen bibliographischen Informationen eines Titels sind hier bereits ein guter Ansatzpunkt. Querverbindungen über Personen, Körperschaften, Schlagworte und Systematiken haben sich bereits seit vielen Jahren durchgesetzt.

Man kann sich nun aber auch weitere Fragen stellen, um thematisch potentiell benachbarte Titel aufzuspüren. Haben die verschiedenen Verfasser des aktuellen Titels beispielsweise mit weiteren Personen in anderen Titeln zusammengearbeitet? Gibt es hier eventuell Häufungen? Diese könnten dem Recherchierenden sicherlich hilfreich sein. Oder, welche anderen Schlagworte kommen bei anderen Titeln zusammen mit den Schlagworten des aktuellen Titels vor? Wie sieht es hier mit Häufungen aus?

Derartige Informationen werden im KUG beim Aufruf eines Titels augenblicklich bestimmt und dem Nutzer als weitere „Entdeckungsmöglichkeit“ über Wortwolken angeboten.

Ebenso lassen sich auch Literaturlisten auswerten, die von Nutzern eingegeben und öffentlich freigegeben wurden. Welche anderen Literaturlisten hat der Nutzer sonst noch erstellt? Könnte da vielleicht etwas Interessantes dabei sein? Kommen Titel der aktuellen Literaturliste vielleicht in anderen Literaturlisten vor? Könnte ich vielleicht hier auf neue Titel stoßen, an die ich bisher noch gar nicht gedacht habe? Auch diese beiden Vernetzungen für Literaturlisten werden im KUG umgesetzt.

Andere Vernetzungen lassen sich aus Nutzungsanalysen bestimmen. Dazu bietet die KUGPlattform eine allgemeine Statistikdatenbank an, in der beliebige nutzungsbedingte Ereignisse abgelegt und später ausgewertet werden können. Nur so erhält die USB Köln als Systembetreiber u.a. diejenigen Informationen, anhand derer sie den Dienst verbessern kann.

Eine sehr wesentliche Information, die aus einer Analyse der KUG-Nutzung gewonnen werden kann, sind Literaturempfehlungen. Die Empfehlungen werden anhand eines speziellen Algorithmus auf Grundlage aufgerufener Vollanzeigen der Titel bestimmt. Um auch hier einen maximalen Nutzen für möglichst viele Kataloge zu erzielen, werden diese Informationen wiederum der zentralen Kataloganreicherung zugeführt.

\section{Weitere Dienstleistungen aus den Daten der KUG-Plattform}

Außerhalb der Universität zu Köln ist der KUG nahezu ausschließlich als Recherche-Plattform bekannt. Einige Bibliotheken nutzen unsere SeeAlso-Dienste, vielfach unbekannt sind jedoch 
andere Dienstleistungen, die wir anhand der in der KUG-Plattform gesammelten Daten lokal an der Universität erbringen.

Wesentlich ist auch hier die lokale Haltung der bibliographischen Informationen in relationalen Datenbanken. Diese Informationen sind so abgelegt, dass sie sich - anders als in vielen Erfassungssystemen - bis auf die Kategorieebene ansprechen lassen.

Allein mit Hilfe dieser Informationen können wir bereits für unsere Bibliotheken als Dienstleistung einen Abgleich zwischen beliebigen Katalogbeständen anbieten. Diese Aufstellung gemeinsamer Titel in den jeweiligen Bibliotheken wird in Form einer CSV-Datei geliefert und ist ein sehr hilfreiches Entscheidungsinstrument für Aussonderungen oder Zusammenlegungen von Bibliotheken.

\section{Beispiel: Bibliotheksführer als E-Book}

Zusätzlich werden in der KUG-Plattform aber nicht nur Katalogdaten abgelegt, sondern auch Informationen über die zugehörigen Bibliotheken. Diese Bibliotheksinformationen werden an der USB Köln in Form eines offiziellen Bibliotheksführers, ehemals in einer aufwändig hergestellten Print-Version, heute online auf den Webseiten geführt. Dabei werden u.a. Adressen, Öffnungszeiten, EDV-Ausstattung, Ansprechpartner, Geo-Koodinaten, Gesamtbestand und vieles mehr erfasst.

Um diese Informationen vollintegriert in der KUG-Anwendung darstellen zu können, werden sie automatisiert aus den Webseiten extrahiert und kategorisiert in den KUG eingespielt.

Es besteht jedoch weiterhin ein großer Bedarf nach einer umfassenden Gesamtübersicht aller Bibliotheken in einer ausdruckbaren Publikation. Mit dem KUG als Datenzentrale, in der alle relevanten Informationen vorliegen, kann die USB Köln einen Bibliotheksführer ${ }^{13}$ im pdfFormat automatisch als weitere Dienstleistung erzeugen. Da die Erzeugung mit keinem Aufwand verbunden ist, wird der ausdruckbare Bibliotheksführer täglich erzeugt und ist entsprechend aktuell. Auch in diesem Prozess finden wieder Mashup-Techniken Anwendung. Anhand der erfassten Geo-Koordinaten einer jeden Bibliothek wird per Mashup mit dem OpenStreetMap Projekt ${ }^{14}$ automatisch ein Lageplan der Bibliothek eingebunden (Abbildung 7).

Da im KUG neben den allgemeinen Informationen über die Bibliotheken gleichzeitig katalogspezifische Informationen verfügbar sind, können aus der Kombination neue Inhalte erzeugt werden. Für den Nutzer wesentlich ist z.B., wieviele Titel des Gesamtbestandes überhaupt elektronisch erfasst und damit recherchierbar sind.

Pro Gruppe an Katalogen - typischerweise fakultätsweise - werden daher die von den Bibliotheken gemeldeten Bestandszahlen zusammen mit der Zahl tatsächlich elektronisch erfasster Titel ausgegeben. Wünschenswert für den Recherchierenden wären diese Angaben über den Erfassungsgrad sicherlich auch bezogen auf jede einzelne Bibliothek. Denn das Hintergrundwissen „Wie sinnvoll ist es eigentlich, im Online-Katalog zu suchen? Oder ist es vielleicht doch besser, den Kartenkatalog zu konsultieren?" ist entscheidend dafür, dass der Recherchierende auch zum gesuchten Medium kommt.

\footnotetext{
13http://kug.ub.uni-koeln.de/bibliotheksfuehrer/. Zuletzt besucht am: 26.3.2010

${ }^{14}$ http://www .openstreetmap.org/. Zuletzt besucht am: 26.3.2010
} 


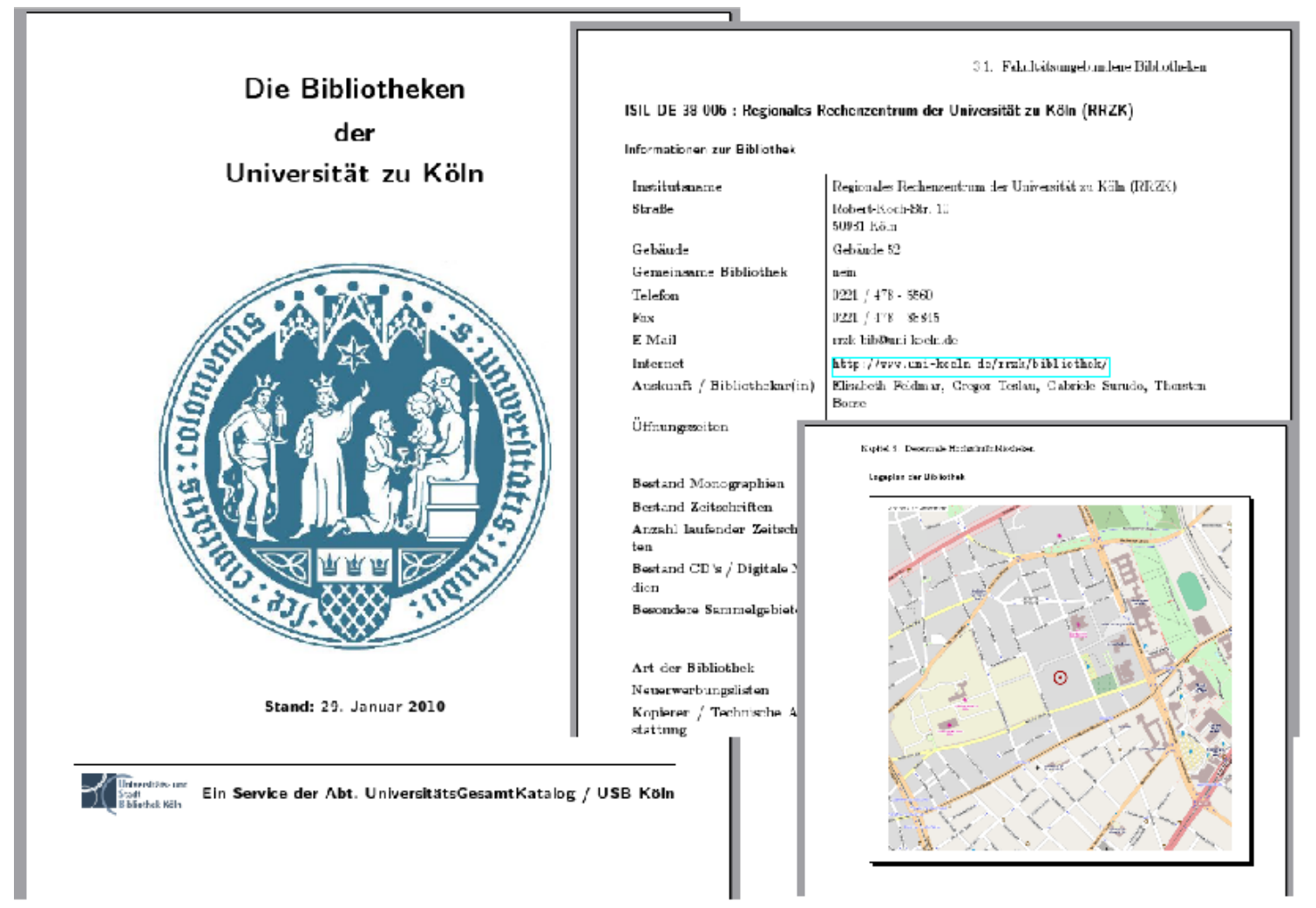

Abbildung 7: Automatisch aus dem KUG erzeugter Bibliotheksführer als E-Book

Die damit verbundene Problematik geht jedoch weit über die einfache technische Realisierbarkeit hinaus.

\section{Beispiel: Liste der Zeitschriften in der ZDB}

An der USB Köln werden zentral die Nachweise der Institutszeitschriften in der ZDB geführt. Als Arbeitsinstrument generieren wir aus dem KUG daher für jedes Institut Listen der Zeitschriften, die aktuell in der ZDB für dieses Institut gemeldet sind. Die Institute können anhand dieser Listen dann überprüfen, ob sich inzwischen Änderungen ergeben haben und uns dies zur Korrektur der Daten in der ZDB zurückmelden.

Als weiteres Angebot an die Institute werden in diesen Listen zur Erwerbungs- und Bestandkoordination automatisch auch zusätzlich Informationen über Parallel-Bestände in anderen Instituten vermerkt. Als allgemeine Information wird am Anfang der Liste neben der Gesamtzahl der Zeitschriften im jeweiligen Institut auch die Zahl der Zeitschriften ausgegeben, die auch in anderen Bibliotheken vorhanden sind. Bei jeder aufgeführten Zeitschrift werden dann konkret diese anderen Bibliotheken anhand ihrer Sigel genannt.

Diese drei Beispiele zeigen, dass sich aus einer zentralen Recherche-Plattform in einem Katalogverbund vielfältige Dienstleistungen für verschiedene Zielgruppen entwickeln lassen, die über die eigentliche Recherche hinausgehen können. 


\section{Neue Möglichkeiten mit offenen Daten}

Schon seit einiger Zeit wird im Bibliothekswesen laut über die Freigabe der bibliographischen Daten als (Linked) Open Data nachgedacht - u.a. in größerem Rahmen bei der Tagung „Semantik Web in Bibliotheken" SWIB0915 im Kontext des Semantic Web.

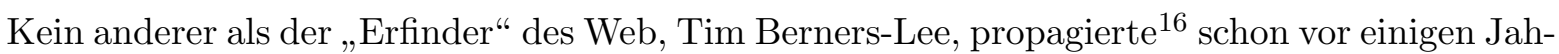
ren die Freigabe von Daten, zunächst als Roh-Daten, dann beschrieben durch Web-Standards, um sie zum integralen Teil des Webs ${ }^{17}$ zu machen. Diese Daten - oder Teile davon - können dann vielfältig genutzt und kombiniert werden - in Anwendungsgebieten, an die man selbst mit seinen Daten eventuell noch gar nicht gedacht hat.

Auch wir in der USB Köln sehen das immense Potential, das sich mit vollständig (gemein)freien bibliographischen Daten erreichen lässt. Um dieses Potential auszuschöpfen, hat die USB Köln zusammen mit verschiedenen anderen Kölner Bibliotheken und dem Landesbibliothekszentrum Rheinland-Pfalz (LBZ) in Kooperation mit dem Hochschulbibliothekszentrum des Landes Nordrhein-Westfalen (hbz) ihre bibliographischen Daten vollständig für die Allgemeinheit geöffnet - in dieser Dimension ein Novum in der deutschen Bibliotheksgeschichte. Allein die USB gab mehr als 3 Millionen Titelsätze frei, zusammen mit den anderen Bibliotheken waren es mehr als 5 Millionen. Wenn weitere Bibliotheken folgen, ließe sich der gemeinsamen Nutzen, den alle aus den Daten ziehen können, noch weiter erhöhen.

Gerade die bereits vorgestellten und in der KUG-Plattform umgesetzten Strategien lassen sich durch Open Bibliographic Data deutlich erweitern und verbessern.

Der Weg hin zu Open Bibliographic Data hat verschiedene Dimensionen ${ }^{18}$, Für den konkreten Einsatz sind, neben der Kostenersparnis durch eine komplette Übernahme von Titeln, vor allem zwei Bereiche wesentlich: Als Quelle für Anreicherungen mit externen Inhalten und zur Verankerung des Bibliotheksbestandes im Netz.

Viele Inhalte aus externen Katalogaufnahmen können zu einer qualitativen Verbesserung der eigenen Daten beitragen. Dazu gehören u.a. Schlagworte, Systematikinformationen wie Basisklassifikation (im GBV und der USB), RVK (im BVB) oder DDC, PND-Nummern, Links zu digitalisierten Inhaltsverzeichnissen mit OCR-Volltexten, katalogisierte freie E-Books und vieles mehr. Mit diesen zusätzlichen Informationen lässt sich - durch zentrale Anreicherung im KUG oder expliziten Import in die einzelnen Kataloge - für den Recherchierenden der Katalogbestand tiefer thematisch erschließen und ein deutlich verbesserter Recherche-Dienst anbieten.

Bibliothekskataloge oder -portale sind nicht mehr die ersten Anlaufstellen, wenn Nutzer heutzutage Informationen zu einem Thema suchen. Schon 2005 ging aus der Studie „Perception of

\footnotetext{
${ }^{15}$ Folien und Videos der Vorträge auf der Tagungsseite http://www. swib09.de/. Zuletzt besucht am: 26.3.2010

${ }^{16}$ Berners-Lee, Tim: The year open data went worldwide http://www.ted.com/talks/view/id/788 . Zuletzt besucht am: 26.3.2010

${ }^{17}$ Dodds, Leigh: Web integrated data http://www.slideshare.net/ldodds/web-integrated-data . Zuletzt besucht am: 26.3 .2010

${ }^{18}$ Pohl, Adrian: Dimensionen von Open Bibliographic Data http://www. uebertext.org/2010/03/dimensionen-von-open-bibliographic-data.html . Zuletzt besucht am: 26.3 .2010
} 
Library and Information Resources $\sqrt{19}$ von OCLC deutlich hervor, dass für 84 Prozent der Recherchierenden Suchmaschinen wie Google die erste Wahl sind. Lediglich 1 Prozent von ihnen beginnen ihre Suche in Online-Datenbanken oder den Webseiten einer Bibliothek.

Um die Nutzer dort abzuholen, wo sie suchen, müssen die bibliographischen Daten mit ihren Bestandsinformationen integraler Teil des Netzes werden. Der einfachste und kostengünstigste Weg ist die Beschreibung dieser Daten in der Sprache des Web mit RDF durch geeigneten Ontologien und Veröffentlichung als Open Bibliographic Data, so dass sie durch Suchmaschinen eingesammelt - und sinnvoll verarbeitet werden können. Auch im lokalen Kontext können sich so Resourcen-orientierte Infrastrukturen Spinellis et al 2009] bilden, die in der Verarbeitung der Informationen neue Perspektiven eröffnen.

Zusätzlich können Querverweise zu anderen Datenquellen, wie z.B. der Dbpedia 20 , LCSH 21 oder STW ${ }^{22}$, eingebracht werden und so die Daten als Linked Open Dat2 ${ }^{23}$ in einem semantischen Web noch wertvoller machen. Durch geeignete Abfragesysteme und Sprachen wie SPARQL lassen sich im Semantic Web durch die Querverbindungen zwischen vielen verschiedenen Datenquellen Fragestellungen beantworten, die so bisher nicht effizient möglich waren, wie in einem Beispiel von Juan Sequeda: „Football Players who went to the University of Texas at Austin, played for the Dallas Cowboys as Cornerback“" 25

\section{Zusammenfassung}

Anhand der KUG-Plattform an der USB Köln wurden verschiedene Beispiele dargestellt, wie durch die besondere Herausforderung vieler, heterogener Kataloge andere Wege beschritten werden mussten als bei einem einzelnen Katalog. Die dabei umgesetzten Lösungen und Prinzipien - Anreicherungen, Mashups und Vernetzungen - sind jedoch so allgemein, dass sie sich auf eine Vielzahl an Arten von Katalogen - einzelne, getrennte oder verteilte - anwenden lassen. Erst durch ihren Einsatz kann für den Recherchierenden im Umfeld heterogener Kataloge ein Höchstmaß an Homogenität erreicht werden.

Speziell die zentrale Anreicherungsdatenbank ist dabei ein zentrales Bindeglied zwischen den ansonsten einzelnen Katalogbeständen im KUG. Sie bietet sich mit den dort enthaltenen Infor-

\footnotetext{
${ }^{19}$ Frage 520: Where electronic information searches begin http://www.oclc.org/reports/pdfs/Percept_all.pdf. Zuletzt besucht am: 26.3.2010

${ }^{20}$ http://dbpedia.org/About. Zuletzt besucht am: 26.3.2010

${ }^{21}$ Library of Congress Subject Headings http://id.loc.gov/authorities/search/. Zuletzt besucht am: 26.3.2010

${ }^{22}$ Standard Thesaurus Wirtschaft http://www.w3.org/2001/sw/sweo/public/UseCases/ZBW/ Zuletzt besucht am: 26.3.2010

${ }^{23}$ Davis, Ian ; Heath, Tom: The thirty minute guide to RDF and Linked Data. http://www.slideshare.net/iandavis/30-minute-guide-to-rdf-and-linked-data Zuletzt besucht am: 26.3.2010

${ }^{24}$ OPENLINK Software: Deploying Linked Data. http://virtuoso.openlinksw.com/Whitepapers/html/vdld_html/VirtDeployingLinkedDataGuide.html Zuletzt besucht am: 26.3.2010

${ }^{25}$ Sequeda, Juan: Introduction to Linked Data. International Semantic Web Conference. 2009 http://www.slideshare.net/juansequeda/introduction-to-linked-data-2341398 Zuletzt besucht am: 26.3 .2010
} 
mationen als „Andockpunkt" für externe Mashups mit dem KUG an, z.B. über den SeeAlsoKonnektor.

Zentral bei der Realisierung und Weiterentwicklung der KUG-Plattform war die Loslösung des Blicks von einem einzelnen Katalog „im Vakuum“ und die Ausweitung auf einen größeren Kontext: Der einzelne Katalog im Verbund mit anderen Katalogen, der Katalogverbund als Teil des Netzes mit vielen Katalogen und Verbünden. Dadurch wurden fast automatisch allgemeine Techniken des Web 2.0 adaptiert, die sich in einem zutiefst heterogenen und schnell wandelnden globalen Netz - dem Internet - in ihrer praktischen Anwendbarkeit durchgesetzt haben.

Niemand kann vorhersagen, welche Dienste oder Daten als nächstes sinnvoll in eine bibliothekarische Rechercheanwendung eingebunden werden können. Auch die Anforderungen an eigene Schnittstellen, über die man zwischen Bibliotheken und Verbünden neue Dienste gemeinsam erschaffen kann, entwickeln sich ständig weiter.

Um mit seinem eigenen Recherchedienst in dieser Zeit des fortwährenden Wandels überhaupt „manövrierfähig“ zu sein, ist die Flexibilität, geeignete Erweiterungen mit möglichst geringem Aufwand selbst vornehmen zu können, so außerordentlich elementar. Dazu bedarf es Systeme, die eine eben solche Flexibilität ermöglichen und die dabei so effizient sind, dass sie den notwendigen Aufwand für Anpassungen, Erweiterungen und Betrieb minimieren.

Der KUG und andere eigenentwickelte Systeme, wie vuFind ${ }^{26}$, Heid ${ }^{27}$, beluga ${ }^{28}$ oder XO$\mathrm{PAC}^{29}$, sind gute Beispiele dafür, den Betreuungsaufwand für eine schwerfällige, proprietäre Lösung sinnvoller in die (Weiter-)Entwicklung und Anpassung bestehender Open Source-Systeme zu investieren.

Dazu kommen weitere Vorteile, denn gerade die beiden damit zusammenhängenden Faktoren - die Auseinandersetzung mit aktuellen und zukünftigen Themen sowie die anschließende selbständige Umsetzung im Rahmen eines vollständig offenen Systems - sind wesentlich für den Aufbau von Wissen und Kompetenz in Bibliotheker ${ }^{30}$.

Während bei einem proprietären System oft nur produktspezifisches Spartenwissen aufgebaut wird, geschieht der Wissensaufbau bei aktuellen, vollständig offenen Systemen in verbreiteten Basistechniken, die sich auch in anderen Projekten sehr gut anwenden lassen und so erst einen wirtschaftlichen und nachhaltigen Betrieb mit begrenzten Finanzmitteln und Personal - für den Nutzer - gewährleisten.

\footnotetext{
${ }^{26}$ http://vufind.org/ Zuletzt besucht am: 26.3.2010

${ }^{27}$ http://www .ub.uni-heidelberg.de/helios/kataloge/heidi.html Zuletzt besucht am: 26.3.2010

${ }^{28}$ http://beluga.sub.uni-hamburg.de/ Zuletzt besucht am: 26.3.2010

${ }^{29}$ http://www. xopac.org/ Zuletzt besucht am: 26.3.2010

${ }^{30} \mathrm{Vgl}$. Analyse von Christensen, Anne ; Christof, Jürgen: beluga - Die Hamburger Rechercheplattform zur Literaturversorgung virtueller Lernräume. 2007.

http://beluga.sub.uni-hamburg.de/blog/wp-content/uploads/2007/10/jourfixe_2007.pdf Zuletzt besucht am: 26.3.2010
} 


\section{Literatur}

[Bauer 2004] Bauer, Delia: Vom zweischichtigen Bibliothekssystem zur funktionalen Einschichtigkeit: Problematik eines Strukturkonzepts am Beispiel der Universitäts- und Stadtbibliothek Köln. Band 43. 2004. Kölner Arbeitspapiere zur Bibliotheks- und Informationswissenschaft.

http://www.fbi.fh-koeln.de/institut/papers/kabi/band.php?key=54

Zuletzt besucht am: 26.3.2010

[Chudnow et al 2006] Daniel Chudnov et al: Introducing unAPI, in: Ariadne. Issue 48 (July 2006)

http://www.ariadne.ac.uk/issue48/chudnov-et-al/

Zuletzt besucht am: 26.3.2010

[Flimm 2007] Flimm, Oliver: Die Open-Source-Software OpenBib an der USB Köln : Überblick und Entwicklungen in Richtung OPAC 2.0. in: BIBLIOTHEK. Forschung und Praxis, Jg. 31 (2007) Nr. 2. http://eprints.rclis.org/9891/

Zuletzt besucht am: 26.3.2010

[Hahn Schulze 2009] Hahn, Ulrich ; Schulze, Matthias: Katalogerweiterungen, Mashups und Elemente der "'Bibliothek 2.0"' in der Praxis. in: Bibliotheksdienst 43. Jg. (2009), H. 1 , S. 20-38

http://www.zlb.de/aktivitaeten/bd_neu/heftinhalte2009/

Erschliessung010109.pdf

Zuletzt besucht am: 26.3.2010

[Spinellis et al 2009] Spinellis et al: Resource-Oriented Architectures : Being "In the Web"'. O'Reilly. 2009. In: Beautiful Architecture - ISBN 978-0-596-51798-4

[Stelzenmüller 2008] Stelzenmüller, Christian: Mashups in Bibliotheken. Untersuchung der Verbreitung von Mashups auf Webseiten wissenschaftlicher Bibliotheken und Erstellung eines praktischen Beispiels. Stuttgart. 2008 http://opus.bsz-bw.de/hdms/volltexte/2008/654/

Zuletzt besucht am: 26.3.2010

[Tonkin et al 2008] Tonkin et al: Collaborative and social tagging networks. in: Ariadne. Issue 54 (January 2008)

http://www.ariadne.ac.uk/issue54/tonkin-et-al/

Zuletzt besucht am: 26.3.2010

[Voß 2007] Voß, Jakob: Tagging, Folksometries and Co - Renaissance of Manual Indexing?. Proceedings of the International Symposium of Information Science.

http://arxiv.org/abs/cs/0701072

Zuletzt besucht am: 26.3.2010

[Voß 2008] Voß, Jakob: SeeAlso : A Simple Linkserver Protocol. in: Ariadne, Issue 57 (October 2008)

http://www.ariadne.ac.uk/issue57/voss

Zuletzt besucht am: 26.3.2010 


\section{Kurz-Biographie des Autors}

Oliver Flimm, Jahrgang 1969, ist Diplom-Physiker und als Administrator sowie Programmierer im Bereich Unix/Linux seit 1991 tätig. Während seines Studiums der Physik mit Nebenfach Informatik arbeitete er an der USB Köln und betreute dort technisch das Projekt InstitutsGesamtKatalog (IGK). Nach Abschluss seines Studiums übernahm er dort als wissenschaftlicher Mitarbeiter neben seiner Tätigkeit im Bereich Unix/Linux-Administration und -Programmierung u.a. die technische Durchführung verschiedener Projekte - insbesondere des KUG-Projektes.

\section{Anschrift des Autors}

\section{Oliver Flimm}

Universitäts- und Stadtbibliothek Köln

Dezernat 1 - EDV

Universitätsstr. 33

D-50931 Köln

E-Mail: flimm@ub.uni-koeln.de

\section{Lizenz}

Der Artikel steht unter der Creative Commons CC-BY Lizenz.

Er wurde veröffentlicht in:

Handbuch Bibliothek 2.0, Hrsg. von Julia Bergman und Patrick Danowski, Berlin, New York: De Gruyter Saur, September 2010, S. 293-316. - ISBN 978-3-11-023210-3 